\title{
MODELOS DE PARTICIPACIÓN SINDICAL EN SUBSIDIARIAS DE EMPRESAS MULTINACIONALES DE ARGENTINA. UN ANÁLISIS SOBRE SUS DETERMINANTES ${ }^{1}$
}

\author{
Sonia Roitter* \\ Analía Erbes** \\ Marcelo Delfini***
}

Resumen. Las empresas Multinacionales (EMN) son actores centrales en el proceso de globalización que con sus operaciones en diferentes partes de mundo y, específicamente, a través de sus prácticas inciden en las instituciones del país de instalación. Las EMN intentan homogeneizar su operatoria a nivel internacional en materia de relaciones laborales y participación sindical, influyendo sobre las lógicas locales y generando dinámicas con configuraciones que van desde la alineación completa con la EMN, hasta el predominio de las instituciones locales en la definición del sistema de relaciones laborales en los espacios de trabajo. En este marco, se proponen dos objetivos centrales: i) identificar diferentes modelos de participación sindical entre las filiales de EMN radicadas en Argentina, y ii) establecer la importancia de factores tales como país de origen, sector de actividad, tamaño, año de ingreso al país, forma de gestión de la fuerza de trabajo y nivel de autonomía del área de recursos humanos frente a la casa matriz en tanto determinantes de los modelos diferenciales mencionados. Para ello se utilizaron dos estrategias analíticas, un análisis de cluster que permitió identificar los diversos modelos de participación sindical y un análisis de regresión logística ordenada para observar la relevancia de los factores condicionantes de los modelos identificados.

Palabras Clave: Gestión del trabajo; Sindicatos; Representación.

\footnotetext{
* Universidad Nacional de General Sarmiento (UNGS), Argentina.

Contacto: sroitter@campus.ungs.edu.ar

** Universidad Nacional de General Sarmiento (UNGS), Argentina.

Contacto: aerbes@campus.ungs.edu.ar

*** Consejo Nacional de Investigaciones Científicas y Técnicas (CONICET), Argentina y Universidad Nacional de General Sarmiento (UNGS), Argentina.
}

Contacto: mdelfini@conicet.gov.ar

1 El presente artículo forma parte de una versión ampliada y desarrollada de la ponencia presentada en la III Reunión iberoamericana de Socioeconomía RISE, Cartagena, Colombia y forma parte de la Investigación "Empresas Multinacionales: Gestión de la fuerza de trabajo y respuestas sindicales 2003-2013" Proyectos de Investigación Científica y Tecnológica FONCyT (Fondo para la Investigación Científica y Tecnológica). PICT (Proyectos de Investigación Científica y Tecnológica) № 2013-0931, financiado por el Ministerio de Ciencia y Tecnología, Argentina. 


\title{
MODELS OF TRADE UNION PARTICIPATION IN SUBSIDIARIES OF ARGENTINA MULTINATIONAL COMPANIES. AN ANALYSIS OF ITS DETERMINANTS
}

\begin{abstract}
Multinational companies (MNCs) are central actors in the globalization process with its operations in different parts of the world and, specifically, through their impact on the institutions in the country of installation. The MNCs attempt to homogenize its operations at the international level in the field of labor relations and trade union participation, influencing the local logical and generating dynamics with configurations ranging from complete alignment with the MNCs, to the predominance of local institutions in the definition of the system of labor relations in the workspace. In this framework, we propose two central objectives: (i) identify different models of union participation among MNCs subsidiaries based in Argentina, and (ii) to establish the importance of factors such as country of origin, sector of activity, size, year of entry in to the country, form of management of the labor force and level of autonomy of the Human Resources area in front of the headquarters; as determinants of the differential models mentioned above. For that, two analytical strategies were used, a cluster analysis that allowed to identify the different models of union participation and an ordered logistic regression analysis to observe the relevance of the conditioning factors to the identified models.
\end{abstract}

Keywords: Work management; Unions; Representation.

Original recibido el 30/10/2018

Aceptado para su publicación el 27/03/2020 


\section{Introducción}

El proceso de globalización tiene a las Empresas Multinacionales (EMN) como uno de sus principales actores. En este sentido, al ingresar a los mercados locales y, a partir de su poder económico, buscan imponer prácticas de empleo y relaciones laborales de carácter homogéneo para el conjunto de sus filiales. Así, las EMN observan a la sindicalización como un fenómeno negativo que va en contra de sus intereses en dos direcciones. En la primera conduciría al aumento de los costos laborales, la protección de los trabajadores, la menor flexibilidad y sensibilidad al cambio y un clima antagónico entre la empresa y los trabajadores (Flood y Toner, 1997) y, en la segunda, sería un factor central que impide u obstaculiza los procesos de homogeneización de gestión de la fuerza de trabajo a nivel global (Gunnigle, Turner y D'Art, 1998).

El poder económico a nivel mundial de las EMN se pone en evidencia cuando se observa que cerca de 100 mil EMN que controlaban 860 mil filiales alrededor del mundo y generaban un valor agregado que representaba el $11 \%$ del PIB mundial en el año 2007(UNCTAD [Conferencia de las Naciones Unidas sobre Comercio y Desarrollo], 2008). La búsqueda por parte de estas empresas de imponer ciertas prácticas de empleo y así homogeneizar sus estilos de conducción y prácticas relativas al trabajo, se enfrenta con las características de las instituciones locales inmersas en una cultura y procesos históricos que le han dado su forma actual, lo que da como resultado diferentes modelos de gestión de la fuerza de trabajo en los países de instalación.

En el caso específico de Argentina, las EMN que operan en la economía local en los sectores de Industria, comercio y servicios son 1.140 y su participación en el empleo representaba 12\% del total del empleo registrado en el año 2011 (Rojo Brizuela, Tumini y Yoguel, 2011). Asimismo, tomando en consideración las 500 firmas más grandes de Argentina, se evidencia una importante participación de firmas extranjeras las cuales explican $79 \%$ del valor agregado generado por este conjunto de empresas en 2013 (Roitter y Erbes, 2017). Esta participación del capital extranjero se ha desarrollado en un contexto caracterizado, históricamente, por una fuerte presencia sindical. Así, las prácticas que buscan imponer las EMN en sus filiales se enfrentan a un modelo de relaciones laborales donde los sindicatos han logrado altos niveles de control sobre las condiciones de trabajo y elevados niveles de afiliación (Delfini, 2011).

De esta manera, entre la búsqueda por homogeneizar su operatoria a nivel internacional y la influencia que ejercen las instituciones locales, se desarrollan procesos que van desde una configuración homogénea de alineación con el conjunto de la EMN, pasando por procesos de hibridación, hasta otros en los que se evidencia el predominio de las instituciones locales para la configuración de las relaciones laborales en los espacios de trabajo.

En este marco, el artículo tiene dos objetivos centrales: en primer lugar, identificar diferentes modelos de participación sindical entre las filiales de EMN radicadas en Argentina y, en segundo lugar, establecer los determinantes que confluyen en la existencia de estos modelos diferenciales, entre los cuales se consideran factores 
tales como el país de origen, sector de actividad, tamaño, año de ingreso al país, forma de gestión de la fuerza de trabajo y nivel de autonomía del área de recursos humanos frente a la casa matriz.

A partir de los objetivos planteados, este artículo busca responder los siguientes interrogantes: ¿qué elementos posibilitan desarrollar un modelo de participación sindical?, ¿cuáles son los modelos de participación sindical emergentes en las EMN?, ¿qué factores permitirían explicar la diversidad de modelos? y ¿cuáles son los indicadores que posibilitan explicar la adopción de los modelos existentes?

Para realizar este análisis se parte de los resultados de una encuesta realizada entre mediados de 2016 y 2017 a 83 filiales de EMN instaladas en Argentina, los cuales fueron abordados con dos estrategias analíticas: un análisis de cluster que permitió identificar los diversos modelos de participación sindical y sus características, y una regresión logística ordenada a partir de la cual se identificaron factores condicionantes de los modelos de participación sindical obtenidos.

El artículo se estructura de la siguiente manera. En la segunda sección se presentan las hipótesis sustentadas en las principales contribuciones conceptuales para la definición de los modelos de participación sindical y sus determinantes. En la tercera sección se desarrolla el análisis empírico a partir de la encuesta mencionada. Finalmente, en la cuarta sección se da cuenta de las principales conclusiones.

\section{Relaciones laborales, presencia sindical y EMN: entre las instituciones locales y las políticas de las empresas}

En términos generales, el sistema de relaciones laborales refiere a un conjunto de regulaciones que trasmutan y que reflejan las relaciones económicas en base a las cuales se determina la correlación predominante entre fuerzas productivas. La existencia de "modelos nacionales" supone que en cada país existe un conjunto específico de elementos que influyen sobre el sistema de relaciones laborales. Entre estos se destacan los cuerpos legales relativos al trabajo, sus relaciones y sus formas de regulación; las tradiciones históricas, y los tipos sindicales de acción e intervención, tanto en el espacio de trabajo como en la vida política y social en general. En este contexto, los sistemas nacionales pueden especificar las normas básicas de las relaciones de trabajo, identificar actores y definir procesos de interacción que contribuyen a estructurar a esos mismos sistemas en base a especificidades culturales, políticas e históricas (Hyman, 1999).

Cuando se analiza específicamente la cuestión de las relaciones laborales y la presencia sindical en las EMN es importante considerar sus motivaciones en el desarrollo de estrategias específicas que se cristalizan en las prácticas de estas firmas hacia los sindicatos. Dicha cuestión fue abordada en el marco analítico de la racionalidad limitada, donde se asume que, por un lado, las firmas multinacionales tratan de optimizar la rentabilidad y así obtener un mayor rendimiento de la fuerza de trabajo mientras que, por el otro, los sindicatos buscan maximizar los beneficios de los trabajadores. Como consecuencia de lo anterior, los directores de las empresas buscan disminuir los niveles de representación sindical siempre que ello implique mejoras en el rendimiento de la operatoria de la filial (Cooke, 2003). Sin embargo, la decisión también puede estar condicionada por el sistema de relaciones laborales 
del país de instalación, sus normas, sus leyes y su historia.

En Argentina, el sistema de relaciones laboralesse basa en un modelo de representación único a nivel de empresa en el que la participación de los trabajadores y de los sindicatos se canaliza a través de los delegados o comisiones internas y que se caracteriza por: 1) una fuerte intervención estatal orientada a la protección, mediante la homologación de los acuerdos y de los convenios colectivos de trabajo, y de la participación en la definición de los actores sindicales; 2) derivado de lo anterior, el fortalecimiento del poder institucional verticalizado del sindicato y del modelo de sindicato único cuya base estructural se asienta en las comisiones internas y el cuerpo de delegados; 3 ) la extensión de la negociación colectiva centralizada por rama de actividad, la definición de la evolución de las remuneraciones en base a la indexación y a la negociación colectiva y la configuración de un amplio sistema de previsión y seguridad social garantizado conjuntamente por el Estado y el sindicato. La conformación por parte de los sindicatos de obras sociales y la presencia sindical en los espacios de trabajo de los representantes fue consolidando altos niveles de afiliación, en tanto estos dos elementos posibilitaron un vínculo más estrecho entre los trabajadores y las organizaciones sindicales.

Durante la década del noventa se introdujeron un conjunto de prácticas flexibilizadoras y antisindicales que, a pesar de la profundidad de los cambios que las sustentaron, tuvieron efectos moderados. Esto se evidencia en el leve descenso que tuvo la tasa de afiliación en el Gran Buenos Aires entre 1990 y 2001 (pasó del 49\% a 42\%) (Marshall y Groisman, 2005), en tanto que para 2005, la tasa de afiliación entre los trabajadores formales de empresas privadas alcanzaba al $41 \%$ y la presencia de los representantes sindicales en el espacio de trabajo se configuraba como uno de los determinantes fundamentales para sostener los niveles de afiliación (Delfini, 2011). La revisión de la literatura y de la evidencia empírica a nivel internacional destacala importancia que adquieren diferentes características de las empresas y de las relaciones laborales en la adopción de prácticas específicas de las EMN hacia los sindicatos. A continuación se retoman estos antecedentes y se plantean las hipótesis particulares para el análisis del caso de las filiales en Argentina.

\subsection{País de origen}

Una parte importante de los estudios sobre EMN se ha centrado en la incidencia que tiene la casa matriz sobre las prácticas de empleo de las subsidiarias, lo cual implica un traslado implícito de las condiciones institucionales desde la primera hacia las segundas superponiéndose, incluso, a las formas institucionales que son las propias de los países receptores. En este marco, una cuestión clave es comprender la intersección entre el comportamiento de la EMN globalizada con la diversidad institucional que se deriva de los sistemas nacionales (Ferner, Edwards y Tempel, 2011; Milcovih y Mitra, 2013).

En general, los estudios que analizan los modelos de vinculación entre empresas y sindicatos destacan que las prácticas hacia los sindicatos en las subsidiarias están alineadas con las estrategias de las casas matrices (Ferner et. al. 2011, Tempel, 2001) por lo que, en tanto las filiales consideren que las prácticas de relaciones laborales desarrolladas desde la matriz son una fuente de ventaja competitiva, 
buscarán imponerlas como parte de su modelo productivo (Barney, 1991). En este marco, por ejemplo, García Calavia (2001) sostiene que la cultura de la empresa estadounidense se ha exportado a otras regiones $y$, entre otras cosas, esto ha derivado en la construcción de un lenguaje empresarial en el que se redefine a los trabajadores como empleados (individuales o en equipos) pero no como parte de colectivos organizados con intereses distintos a los de la empresa. De esta forma se argumenta que el sistema de relaciones estadounidense es diferente al de otros países desarrollados, hablándose de un "excepcionalismo americano" (Jacoby, 1985).

Sin embargo, otros estudios sostienen que las características del sistema en el que se insertan las filiales de EMN cuando se radican en otros países les aporta a estas ciertos márgenes de libertad con respecto a la matriz para introducir prácticas diferenciales adaptadas al contexto nacional que las recibe. En particular, estudios sobre la presencia de empresas de EEUU e Inglaterra en Alemania, donde regulación está vinculada a la fortaleza sindical en los espacios de trabajo, aportan evidencia en esta dirección (Royle, 1998; Tempel, 2001).

Hipótesis 1. El modelo de participación sindical adoptado en las filiales de Argentina está fuertemente influido por el país de origen de la EMN. En particular, se espera que en las firmas provenientes de EEUU tienda a predominar un modelo caracterizado por menores niveles de participación sindical.

\subsection{Actividad económica}

Los análisis desarrollados en países de Europa ponen de manifiesto la importancia de observar al sector como un rasgo explicativo del modelo de las relaciones laborales. Estas investigaciones que adoptan una metodología comparativa entre sistemas consideran que este es un factor central para explicar la divergencia o convergencia de modelos (Katz y Darbishire, 2000; Marginson y Sisson, 2004; Marginson, Arrowsmith y Gray, 2008) y, en particular, trabajos como el de Bechter, Brandly Meardi (2012) señalan que son las lógicas sectoriales, más que las cuestiones nacionales, las que le imprimen rasgos específicos a las relaciones laborales. Como consecuencia de lo anterior, se sostiene que existen más diferencias entre sectores al interior de un mismo país que entre países, lo cual permite establecer diferentes tipos de relaciones laborales organizados en torno a la actividad económica, aún en el marco de un mismo sistema nacional de relaciones laborales (Meardi, 2004).

En esta dirección análisis sobre el sector de actividad de las empresas y la densidad sindical han observado una relación positiva entre la pertenencia al sector industrial y el nivel de afiliación, aunque se ha advertido que la importancia de este factor se redujo en los últimos años (Charlwood y Haynes 2008; Machin 2004). Este mayor arraigo de los sindicatos en las actividades manufactureras puede vincularse con dos cuestiones. Por un lado se argumenta que en estas actividades existe mayor propensión a la afiliación como consecuencia de la distinción que se produce en el desarrollo de las mismas entre los gerentes y los trabajadores operativos, lo cual aparece mucho más diluido en los sectores de comercio y servicios y condiciona negativamente el desarrollo de una identidad intragrupo (Haberfeld 1995; Riley 1997). Por otro lado, se sostiene que la mayor presencia sindical no se vincula 
con el sector en sí mismo, sino con los perfiles y categorías ocupacionales que se emplean. La importancia relativa que adquirió el sector servicios a partir de la década de los ochenta trajo consigo importantes modificaciones, en tanto se concentró en la demanda de perfiles laborales de alta y baja calificación que son, de acuerdo a lo que establece la literatura, los menos propensos a sindicalizarse (Hyman, 1996).

En este contexto, entre otros, es interesante destacar el estudio realizado por Katz y Darbishire (2000) quienes, a partir de la comparación entre el sector metalúrgico y el de telecomunicaciones en siete países en el marco de su teoría de "divergencias convergentes" observaron la convergencia entre sectores pero una divergencia entre países.

Hipótesis 2. Los modelos de participación sindical en las filiales están influidos por el sector de actividad. En este sentido, se espera que en el sector industrial estén sobre-representados aquellos modelos que implican una mayor participación sindical con respecto a las actividades de comercio y servicios.

\subsection{Año de instalación de la filial}

Algunos estudios han señalado que el año de instalación de las filiales constituye un elemento importante de diferenciación entre las estrategias adoptadas hacia los sindicatos. En particular, observan que entre las empresas de nueva instalación predominaban acciones orientadas a evitar la intervención sindical en los espacios de trabajo. A esto se suma que las firmas de radicación reciente concentran sus actividades en la producción de servicios y, más específicamente, en sectores que no presentan tradición sindical, por lo que los empleados muestran una cierta reticencia a la participación sindical.

En este marco, y con el efecto complementario de las reformas liberales es posible sostener que las firmas de nueva instalación y que están vinculadas con nuevos sectores de alta tecnología buscan, a nivel mundial, mecanismos para minimizar o incluso evitar la representación gremial (Gunnigle, Lavelle y McDonnell, 2007).

En relación a ello, algunos artículos resaltan la importancia que ha cobrado en Irlanda la práctica denominada doublebreasting, surgida en la industria de la construcción en EE.UU. que se trasladó a otros sectores y países. Básicamente, el doublebreasting consiste en una participación sindical diferencial entre establecimientos de una misma firma que se traduce, incluso, en la ausencia de representación sindical en algunos de ellos. Este formato, dio lugar a la apertura de nuevos establecimientos con el fin de imponer un formato de representación que pudiera escapar a las organizaciones sindicales (Gunnigle et al., 2007).

Hipótesis 3. El modelo de participación sindical está determinado por el momento de instalación de la filial de la EMN. Así, se espera que en aquellas subsidiarias instaladas desde principio de los años noventa prevalezcan modelos caracterizados por una menor participación sindical.

\subsection{Tamaño de las empresas}

Diversos aportes en la literatura reconocen heterogeneidades en los modelos de relaciones laborales aplicados a empresas de diferente tamaño relativo. Así, por ejemplo, Trebilcock (1998) señala que el tamaño es una cuestión central en la 
definición de la estrategia de relaciones laborales adoptada por una firma y, en este sentido, lo que tiende a predominar en las empresas más pequeñas son esquemas de relaciones informales con los trabajadores, en un contexto de escasa o nula presencia de los sindicatos y otras formas colectivas de representación. El análisis específico sobre subsidiarias de empresas multinacionales está menos presente en los estudios revisados, aunque tampoco se identifican elementos que permitan discriminar el comportamiento de empresas locales y filiales en lo que hace a la relación entre modelos de representación y tamaño de firma.

En este marco, la revisión de distintas experiencias nacionales pone de manifiesto que la relación positiva entre tasa de sindicalización y tamaño de la firma se asocia con el cumplimiento por parte de las EMN de regulaciones específicas establecidas por los sistemas de relaciones laborales nacionales, antes que con una estrategia definida por estas mismas filiales (Hyman, 1999; Cruz Villalón, 2003). En general, los sistemas de relaciones laborales nacionales son los que establecen la cantidad y el mínimo de delegados en función de los ocupados totales que posee la firma. En el caso particular de Argentina, esto se traduce en la posibilidad de contar con delegados en empresas que tienen más de 10 ocupados, mientras que la afiliación, de carácter voluntario, solamente comprende a los asalariados formales, es decir, a los trabajadores registrados por sus empleadores.

Asimismo, se observa que la menor presencia sindical tiende a ser un rasgo característico no entre las empresas de menor tamaño relativo per se, sino entre aquellas que tienen un menor número de empleados y que realizan actividades comerciales y de servicios (Hyman, 1999), por lo que en muchos casos es necesario considerar el tamaño y el sector como variables complementarias para comprender la dinámica de las relaciones laborales.

Hipótesis 4. El modelo de participación sindical adoptado por las filiales de EMN en Argentina está condicionado el tamaño de las empresas. En particular, la participación será mayor a medida que aumente la cantidad de ocupados de la subsidiaria y, a su vez, cuando esta desarrolle actividades vinculadas con la producción manufacturera.

\subsection{Control y autonomía en las filiales de EMN}

Otro de los elementos que configuran las relaciones laborales en las subsidiaras de las EMN es el nivel de control ejercido por la casa matriz y, en ese marco, la autonomía que tiene la filial para avanzar sobre lógicas diversas de relación con los sindicatos (Ferner y Almond, 2013).

Al respecto, Martínez y Jarillo (1989) distinguen entre mecanismos más informales y sutiles de control y mecanismos estructurales y formales. Estos últimos implican monitorear el desempeño de las filiales en diferentes cuestiones en torno al empleo (plantilla, productividad y actitudes de los empleados, entre otras) y, durante los últimos años, su implementación se ha profundizado a través de las posibilidades que brindan las herramientas informáticas para ejercer el control en tiempo real. En esta dirección, Ferner et al. (2011) destacan la importancia de la estructura de la función internacional de recursos humanos para explicar las variaciones en el nivel de control central y en la autonomía de las subsidiarias y, en ese marco, el grado de integración del área de recursos humanos a su semejante en la casa matriz permite 
da cuenta del grado de control que ejerce esta última sobre las políticas de recursos humanos implementadas por las filiales.

Tanto el grado de integración productiva de la filial, como la estrategia de negocios desarrollada por la EMN condicionan los niveles de control que ejerce la casa matriz sobre la subsidiaria. Por una parte, mayores niveles de integración de la filial con la matriz opera en pos de mayores niveles de control de esta última sobre la primera, lo cual restringe la autonomía de las filiales para la adopción de distintas formas de gestión de la fuerza de trabajo (Harzing 1999; Child, 2001; Harzing, 2001). La estandarización de las actividades complementa la dinámica anterior: cuanto mayor sea la estandarización a nivel mundial, tanto más tenderán a estandarizarse las funciones de recursos humanos (Edwards et al., 2007). Por otra parte, las diferentes estrategias globales de negocios de las EMNs conllevan esquemas de control específicos. Así, por ejemplo la literatura sobre EMN indica que la forma de ingreso a los mercados locales (compra o fusión versus inversiones greenfield) condicionará la forma de organización de la nueva empresa: mientras que en el primero de estos casos la subsidiaria se organizará sobre la base de estructuras preexistentes que tenderán a resistir dinámicas de control a partir del retraso en la incorporación de nuevas prácticas de gestión, en las inversiones greenfield existe un mayor espacio para la implementación de sistemas de control que responden a las necesidades del contexto institucional en el que se desarrollan las casas matrices (Edwards, Ress y Coller, 1999).

Una parte importante de esta literatura sostiene que existen rasgos institucionales que generan cierto margen de autonomía a la filial más allá del nivel de integración y control que busca ejercer la casa matriz (Bélanger, Giles y Grenier (2003); Kristensen y Zeitlin 2005). Así, el contexto institucional se vislumbra como una herramienta para resolver la tensión existente entre la dimensión global de la EMN y la dimensión local. El sistema de relaciones laborales nacional puede restringir la imposición de prácticas de gestión de la fuerza de trabajo desde la casa matriz (Edwards y Kuruvilla 2005) y, de esta manera, relajar los niveles de control que ejercen estas sobre las subsidiarias locales (Edwards, Marginson y Ferner, 2013).

Hipótesis 5a. La adopción de un modelo de participación sindical en la firma está condicionado por el nivel de autonomía que presenta la filial local.

Hipótesis $5 b$. El grado de integración de la gestión de la fuerza de trabajo de la filial a niveles superiores de la EMN condiciona el modelo de participación sindical adoptado por la subsidiaria.

\subsection{Individualización salarial}

Existe literatura donde se sostiene que, al tratar de evadir la presencia sindical en las EMN, estas firmas han adoptado ciertas formas de gestión de la fuerza de trabajo como estrategias antisindicales. Estas prácticas que buscan dominar el escenario en la empresa se asocian principalmente con formas de individualización de las prácticas de empleo que se contraponen a las relaciones colectivas de trabajo. Entre estas prácticas individualizantes los sistemas de pagos por rendimiento (SPR) ocupan un rol central. Básicamente se trata de la incorporación de los niveles operativos de las empresas en los esquemas de incentivos utilizados para directores 
y mandos medios. Estos sistemas, sobre todo aquellos basados en la evaluación de desempeño, tienen como objetivo imponer una diferenciación salarial entre los mismos trabajadores que está sujeta a desempeño y/o resultados (Marginson et al., 2008) y reflejan una orientación esencialmente individualista que excluye o limita la participación sindical (Gunnigle, 1995). En general se distinguen tres formas de SPR: a) programas de remuneración individual; b) los incentivos que establecen un vínculo entre la remuneración y la producción o venta de los trabajadores, y c) esquemas situados a nivel de la organización. Éstos últimos suelen ser anuales y de menor importancia cuantitativa que los anteriores (Van Het Kaar y Grünell, 2001). En su asociación con las características asumidas por las relaciones laborales se ha encontrado que los SPR vinculados a las dos primeras formas (a y b) han impactado sobre la influencia de los sindicatos en la determinación de los salarios y erosionado la relación entre los representantes sindicales y los trabajadores, limitando la capacidad de movilización de los sindicatos (Heery, 1997, 2000; Marginson et al., 2008). En este sentido, los programas de remuneración individual vinculados a evaluaciones de desempeño representan la principal amenaza para el accionar de los sindicatos (Arrowsmith y Marginson, 2011), seguido por los planes SPR asociados a incentivos basados en la productividad (forma b).

En este mismo orden, la evidencia indica que las formas de SPR que se introducen para mejorar el control sobre el proceso de trabajo son más propensas a contraponerse a los controles sindicales y, por lo tanto, a socavar la influencia de estos, que aquellas otras formas que están diseñadas para servir a una función de mercado (Gunnigle et al., 1998). No obstante, en algunas empresas de Argentina, los procesos de evaluación de rendimiento y pagos variables, fueron incorporados a los Convenios Colectivos de Trabajo (CCT) negociados con los sindicatos.

Hipótesis 6a. La adopción de modelos de participación sindical está condicionada por formas de evaluación de desempeño. Así, una evaluación de carácter no individualizante se asocia con mayores niveles de participación sindical

Hipótesis 6b. En la misma dirección, los pagos por rendimiento atados a productividad u objetivos se vinculan con menores niveles de participación sindical.

\subsection{Organización del trabajo}

En los últimos años se desarrollaron un conjunto de transformaciones en la dinámica del proceso productivo que estuvieron orientadas a lograr una mayor flexibilidad de respuesta de las firmas ante los cambios producidos en el entorno. En el plano microeconómico, esto se tradujo en el énfasis puesto en la gestión de la calidad, la reducción permanente de costos, tiempos y stocks y la introducción de nuevas tecnologías en la producción. A su vez, todo esto dio lugar a cambios en distintos aspectos organizativos, entre lo que se destacan el trabajo en equipo, la polivalencia y la participación de los trabajadores en distintas instancias formuladas por las empresas (Linhart,1997). De la combinación entre estos aspectos surgen nuevas formas organizacionales, todas ellas distintas al sistema fordista, pero que comparten como rasgo característico la posibilidad de aumentar los niveles de productividad y de generar un mayor grado de flexibilidad en las empresas.

Estas nuevas formas organizacionales muestran una relación dual con los modelos de 
participación sindical (Delfini, 2011). Así, puede observarse una resistencia por parte de los sindicatos a la incorporación de las formas más novedosas de organización del trabajo, en tanto que, a través de estos cambios tiende a incrementarse el rendimiento de la fuerza de trabajo sin que esto se traduzca en beneficios directos para los trabajadores. Como consecuencia de esto, las formas organizacionales con un mayor nivel de flexibilidad son especialmente factibles en contextos de menor presencia y fuerza sindical en los espacios de trabajo. No obstante, y en la medida que los sindicatos van incorporando y aceptando la presencia de ciertos elementos vinculados con estas formas organizacionales (por ejemplo, la rotación entre puestos), comienza a generarse un proceso de dualización en el que no necesariamente niveles altos de flexibilidad se corresponden con debilidad sindical (Delfini, 2011).

Hipótesis 7. Las formas de organización del trabajo flexibles se desarrollan en espacios de trabajo con modelos de participación sindical que implican una menor presencia y representación de estos actores.

\section{La evidencia empírica sobre las filiales de EMN en Argentina}

Para analizar empíricamente las hipótesis planteadas se consideró una base de datos que reúne información sobre 83 filiales de EMN en Argentina, pertenecientes a diferentes sectores de actividad. Se considera como EMN a aquellas que desarrollan actividades productivas en, al menos, un país adicional a aquel en el que se asienta su casa matriz y, en este caso en particular, se consideran empresas cuya casa matriz se encuentra fuera de Argentina. Como marco muestral para este estudio se consideró un padrón de empresas multinacionales con filiales en el país, construido a partir del registro de las filiales y subsidiarias locales de las EMN con actividad en Argentina que surge de listados de empresas entregados por las cámaras de comercio bilaterales. A partir de esta información se elaboró una muestra no probabilística de 300 casos, y se obtuvo respuesta de un grupo de 83 empresas que respeta las cuotas de actividad y tamaño proporcionales a los subgrupos de la muestra original y que poseen más de 20 empleados $^{2}$. El relevamiento fue realizado a través de entrevistas presenciales entre fines de 2016 y principios de 2017 , fundamentalmente en la Provincia de Buenos Aires y las ciudades de Buenos Aires y Córdoba, Argentina. El mismo fue realizado en el marco del PICT N ${ }^{\circ}$ 2013-0931, con sede en la Universidad Nacional de General Sarmiento (UNGS), Argentina.

Dada la relevancia central que en este trabajo adquieren los diversos modelos de participación sindical de las EMN se recurrió, en primer lugar, a construir un indicador que permita captar los diferentes modelos y sus rasgos sobresalientes, a través de un análisis factorial de correspondencias múltiples y de cluster. Por su parte, para estudiar la relevancia de los factores que condicionan la adopción de cada uno de los modelos identificados se recurrió a un análisis de regresión logística ordenado.

Si bien la noción de participación sindical refiere a diversos factores vinculados con la negociación, el conflicto y diversas instancias de acción de los sindicatos,

2 Cabe aclarar que, según el Ministerio de Trabajo, Empleo y Seguridad Social de la Nación (MTEySS), la población de filiales en Argentina llegaba en 2015 a 1.140 en los sectores de industria, comercio y servicios. 
a los fines de este artículo esta dimensión se vincula con el despliegue sindical al interior de la firma en lo relativo al nivel de penetración que logran los sindicatos. Es por esto que los elementos centrales para evaluar la participación sindical en este trabajo están asociados con la presencia de delegados, de comisiones internas y/o de cuerpos de delegados, y con el grado de afiliación, lo que permite dar cuenta de la capacidad de negociación al interior de la empresa. Como consecuencia de lo anterior, la construcción del indicador de modelo de participación sindical considera simultáneamente las siguientes tres dimensiones:

- el nivel de afiliación sindical de los empleados del área de mayor cantidad de ocupados (AMNE), el cual asume tres categorías posibles: no posee personal afiliado, posee hasta un $50 \%$ de afiliados sobre el total del AMNE o posee más de un $50 \%$ en esa condición;

- la presencia de delegados en la filial, con dos categorías, posee o no posee; - la política gerencial hacia los representantes sindicales en la firma, que considera la actitud de la empresa hacia el sindicato (negocia o resuelve) en el tratamiento de cuestiones relativas a salarios, categorías profesionales, jornadas y pausas en el trabajo y esquemas de pagos variables. De aquí surgen tres categorías de acuerdo al grado de negociación prevaleciente: no negocia (la filial decide unilateralmente sobre todas las cuestiones); negocia en casi todos los aspectos mencionados (se negocia en tres aspectos y se decide unilateralmente en uno); negocia algunos (situación intermedia entre las dos anteriores).

A partir de ello se realizó un análisis factorial de correspondencias múltiples (AFCM) y un análisis de cluster con la finalidad de definir tres modelos de participación sindical. El AFCM es una técnica que se enmarca dentro de las estadísticas descriptivas multidimensionales y tiene por finalidad, a través de la reducción de la dimensionalidad del fenómeno estudiado, extraer los principales factores que reflejan la variabilidad de un conjunto de datos y las vinculaciones entre variables y sus categorías. De esta manera, es posible reducir el número de variables con las cuales se trabaja, facilitando el análisis de la información (Roitter, 1991 y Crivisqui, 1993). A partir del AFCM se analizan todas las asociaciones existentes entre las diferentes modalidades de las variables que componen una matriz de datos y, de esta manera, se obtiene un conjunto de clases compuestas por EMN que presentan una alta homogeneidad intragrupo y una elevada heterogeneidad extragrupo.

Tomando la información de los primeros diez ejes factoriales del AFCM, se realizó el análisis de clustery, como resultado de ello, se obtuvieron tres grupos de EMN que, al interior, poseen características similares. Esto es así porque los agrupamientos resultantes se conforman con las empresas que son más cercanas en términos de las distancias euclidianas calculadas con las coordenadas de posicionamiento de los individuos en relación con los ejes factoriales considerados.

Una de las principales ventajas de esta metodología es que realiza una clasificación básicamente empírica (Fernández Macías, 2004). A su vez, este método presenta dos ventajas adicionales con respecto a la construcción "manual" de los grupos. En primer lugar, incluye a todos los individuos para los cuales pueda estimarse al 
menos uno de los indicadores considerados como variables activas, de manera tal que ninguno queda sin incluir en los grupos conformados. En segundo lugar, se trata de una técnica que minimiza la manipulación de los datos y, en este sentido, aporta mayor objetividad a la construcción y a la definición de categorías.

Tal como se muestra en la Tabla 1, cada uno de los tres grupos obtenidos de acuerdo a los modelos de participación sindical se asocia claramente a las diferentes categorías de las variables activas consideradas. Así, el modelo que se denomina "baja participación" se define porque: i) en un $86 \%$ de los casos su personal no está sindicalizado (frente a un 30\% para la media muestral); ii) en la totalidad de los casos no existen delegados (frente al $35 \%$ para la media), y iii) en un $90 \%$ de los casos la EMN no negocia ni con los trabajadores ni con el sindicato cuestiones referidas a salarios, categorías, jornadas y pagos variables (versus $43 \%$ de la media). El segundo modelo, de representación media, se define, en todos los casos, por la presencia de personal sindicalizado que, como máximo, da cuenta del $50 \%$ del AMNE de la empresa. A su vez, en este grupo un $94 \%$ cuenta con presencia de delegados y en un $53 \%$ de las empresas se negocia al menos algunos aspectos con el sindicato (frente al $31 \%$ promedio). Finalmente, el cluster de alta representación posee, en todos los casos, más del $50 \%$ de su personal afiliado a un sindicato y, también en todos los casos, existen delegados en las empresas.

\section{Tabla 1. Caracterización de los modelos de participación en función variables activas}

\begin{tabular}{|c|c|c|c|c|c|}
\hline \multirow{2}{*}{ Variable } & \multirow{2}{*}{ Categoría } & \multirow{2}{*}{$\begin{array}{l}\text { Media } \\
\text { muestral }\end{array}$} & \multicolumn{3}{|c|}{ Modelo de participación sindical } \\
\hline & & & Bajo & Medio & Alto \\
\hline \multicolumn{3}{|c|}{ Proporción de firmas en cada grupo } & $35 \%$ & $20 \%$ & $45 \%$ \\
\hline \multirow{3}{*}{$\begin{array}{c}\text { Nivel de } \\
\text { sindicalización }\end{array}$} & $0 \%$ & $30 \%$ & $86 \%$ & - & - \\
\hline & Hasta $50 \%$ & $20 \%$ & - & $100 \%$ & - \\
\hline & Más de $50 \%$ & $50 \%$ & - & - & $100 \%$ \\
\hline \multirow{2}{*}{$\begin{array}{l}\text { Presencia de } \\
\text { delegados }\end{array}$} & Ausencia & $36 \%$ & $100 \%$ & & \\
\hline & Presencia & $44 \%$ & & $94 \%$ & $100 \%$ \\
\hline \multirow{3}{*}{$\begin{array}{l}\text { Política de } \\
\text { negociación }\end{array}$} & Decide sola & $43 \%$ & $90 \%$ & - & - \\
\hline & Negocia algunos & $31 \%$ & - & $53 \%$ & - \\
\hline & Negocia casi todos & $26 \%$ & - & - & - \\
\hline
\end{tabular}

Fuente: Elaboración propia en base a encuesta a EMN.

Dado que la variable dependiente (modelo de participación) puede asumir tres valores en orden creciente de importancia, la verificación de la relación requiere la utilización de un modelo con datos ordenados estimado mediante el método de máxima verosimilitud. En este caso se optó por la utilización de un modelo de regresión logística ordenado (OLOGIT) en los que, a diferencia de los modelos de regresión probabilísticos ordenados (OPROBIT) que suponen la existencia de una distribución normal de los errores, está implícita la distribución logística de los mismos. 
En lo que respecta a la interpretación de los coeficientes $\beta$ arrojados por este tipo de modelos, es importante destacar que los cambios en la variable dependiente como consecuencia de las modificaciones en el conjunto de variables independientes no pueden interpretarse de manera directa como en el caso de los modelos de regresión lineal. Sin embargo, es posible considerar tanto la significatividad como el signo de estos coeficientes para obtener una medida de la importancia de las variables independientes en la explicación de la variable dependiente.

Complementariamente, el análisis de efectos parciales permite predecir cómo cambia la probabilidad de poseer un modelo u otro de participación sindical al modificarse alguna de las variables independientes relevantes que surgen de la estimación del modelo. Estos efectos no son constantes, sino que dependen de los valores de las demás variables para las cuales generalmente se toman como referencia los valores medios.

Las variables independientes en este modelo de regresión logística ordenado son las siguientes: i) sector de actividad (industrial versus resto); ii) país de origen (otros países versus Estados Unidos); iii) año de inicio de actividades en Argentina (hasta 1989 versus posterior); iv) pagos variables (sin adicionales versus con adicionales); v) estrategia de evaluación de desempeño (no individualizante versus individualizante) ${ }^{3}$; vi) grado de integración del área de $\mathrm{RRHH}$ de la subsidiaria con la casa matriz (no integrada versus integrada); vii) forma de organización del trabajo (tradicional versus moderna) ${ }^{4}$; viii) grado de autonomía con respecto a la casa matriz para decisiones de selección de personal, salariales y de ascenso (autónoma versus resto); ix) tamaño. Con excepción del número de ocupados que es continua el resto de las variables independiente son categóricas: asumen valor 1 cuando, según las hipótesis planteadas, estas se asocian positivamente con modelos de mayor participación sindical y 0 en caso contrario.

Tal como surge de la Tabla 2, las variables que afectan significativamente $(P<0,10)$ y de manera positiva al modelo de participación sindical son el sector de actividad industrial, la instalación en el país hasta 1989, las estrategias no individualizantes y el número de ocupados en la filial local.

A partir de lo anterior, se tomaron las variables independientes significativas en la estimación del modelo para realizar un análisis de efectos parciales. A partir de este, es posible dar cuenta de la probabilidad de pertenencia de una filial a cada modelo de participación cuando se modifica de manera individual alguna de las variables categóricas significativas, se deja en 0 (cero) al resto de los indicadores significativos y en el valor medio a las variables no significativas para la explicación del modelo estimado. Complementariamente, se consideraron diversas combinaciones en los

3 Se considera no individualizante cuando, o bien no existe un sistema de evaluación de desempeño, o si el mismo existe, sus resultados no inciden ni en las remuneraciones ni en las decisiones sobre ascensos y cambios de categoría, a diferencia de la individualizante.

4 La organización del trabajo es llamada tradicional cuando predomina el trabajo individual, o en equipos con predominio de actividades de ejecución frente a las de planificación, no existen planes de rotación para el personal y los trabajadores tienen escasos canales de participación en la toma de decisiones. 
valores de las variables relevantes, con el fin de observar el impacto que tiene cada uno de los factores individual o grupalmente en la configuración de los modelos de participación estudiados.

Tabla 2. Resultados de estimación de modelo logístico ordenado

\begin{tabular}{|c|c|c|c|c|c|}
\hline $\begin{array}{c}\text { Modelo de } \\
\text { representación }\end{array}$ & Coeficiente & $\mathbf{X}$ & $\mathbf{P}>|\mathbf{z}|$ & \multicolumn{2}{|c|}{ Intervalo de confianza } \\
\hline Industria & 2,57782 & 4,15 & 0,000 & 1,360871 & 3,794771 \\
\hline No USA & 0,97000 & 1,61 & 0,107 & $-0,2059025$ & 2,099283 \\
\hline Hasta 1989 & 1,05817 & 1,73 & 0,083 & $-0,1376875$ & 2,254025 \\
\hline Sin adicionales & 0,40839 & 0,67 & 0,504 & $-0,7905125$ & 1,607292 \\
\hline $\begin{array}{c}\text { No } \\
\text { individualizante }\end{array}$ & 1,26934 & 2,07 & 0,038 & 0,0675862 & 2,471084 \\
\hline No integrada & 0,14237 & 0,26 & 0,791 & $-0,9120925$ & 1,196823 \\
\hline Tradicional & 0,08748 & 0,15 & 0,881 & $-1,059764$ & 1,234725 \\
\hline Autónoma & $-0,37896$ & $-0,59$ & 0,552 & $-1,628663$ & 0,8707349 \\
\hline $\begin{array}{c}\text { Tamaño } \\
\text { (ocupados) }\end{array}$ & 0,00028 & 2,18 & 0,029 & 0,0000287 & 0,0005347 \\
\hline
\end{tabular}

Fuente: Elaboración propia en base a encuesta a EMN

Los resultados encontrados se muestran en la Tabla 3. Se parte de una situación inicial en la que las empresas desarrollan actividades comerciales y/o de servicios, se instalaron con posterioridad a 1989 y llevan a cabo prácticas individualizantes. Luego, se presenta la modificación individual de los valores de las tres variables categóricas relevantes para explicar la relación. Así, por ejemplo, en todos los casos la probabilidad de pertenecer a un modelo de baja participación es elevada y la excepción fundamental la constituyen las empresas dedicadas a las actividades manufactureras, donde la probabilidad más elevada se evidencia en relación con el modelo de mayor participación sindical. De esta manera, desarrollar actividades comerciales o de servicios aumenta claramente la probabilidad de pertenecer a un modelo de baja participación sindical cuando se lo compara con las filiales del sector manufacturero e independientemente de los valores asumidos por las restantes variables.

Luego, entre la quinta y séptima fila se muestran los cambios en probabilidad de pertenencia a cada grupo al modificar de a dos los valores de las variables categóricas. En este caso, la probabilidad de pertenecer al grupo de mayor participación aumenta claramente cuando se combina el formar parte del sector manufacturero con haber ingresado al país previamente a los 90 s aun cuando se trate de organizaciones que llevan a cabo estrategias individualizantes, o cuando se trata de empresas industriales que han ingresado con posterioridad a 1990 pero en las que no predominan estrategias individualizantes. Finalmente, la última fila pone de manifiesto que cuando se trata de empresas industriales, instaladas con anterioridad a los noventa y que no desarrollan estrategias individualizantes la 
probabilidad de formar parte de modelos de alta participación sindical alcanza a un $90 \%$, frente a solamente un $6 \%$ que se logra cuando las firmas adquieren las características inversas (primera fila de la Tabla 3).

Tabla 3. Efectos parciales. Estimación de probabilidad de pertenencia a cada modelo.

\begin{tabular}{|c|c|c|c|c|c|}
\hline \multicolumn{2}{|c|}{ Variables significativas } & \multicolumn{2}{c|}{$\begin{array}{c}\text { Probabilidad de pertenencia } \\
\text { a modelos de participación } \\
\text { sindical (\%) }\end{array}$} \\
\hline Actividad & $\begin{array}{c}\text { Año de } \\
\text { ingreso al país }\end{array}$ & $\begin{array}{c}\text { Evaluación de } \\
\text { desempeño }\end{array}$ & Baja & Media & Alta \\
\hline $\begin{array}{c}\text { Servicios y } \\
\text { comercio }\end{array}$ & Desde 1990 & Individualizante & 77 & 17 & 6 \\
\hline Industria & Desde 1990 & Individualizante & 20 & 32 & 48 \\
\hline $\begin{array}{c}\text { Servicios y } \\
\text { comercio }\end{array}$ & Hasta 1989 & Individualizante & 53 & 30 & 17 \\
\hline $\begin{array}{c}\text { Servicios y } \\
\text { comercio }\end{array}$ & Desde 1990 & No individualizante & 48 & 32 & 20 \\
\hline Industria & Hasta 1989 & Individualizante & 8 & 20 & 72 \\
\hline $\begin{array}{c}\text { Servicios y } \\
\text { comercio }\end{array}$ & Hasta 1989 & No individualizante & 25 & 34 & 41 \\
\hline Industria & Desde 1990 & No individualizante & 7 & 17 & 76 \\
\hline Industria & Hasta 1989 & No individualizante & 3 & 7 & 90 \\
\hline
\end{tabular}

Fuente: Elaboración propia en base a encuesta a EMN

Por último se consideró la importancia del tamaño de las EMN en la adopción de cada modelo de participación sindical, a partir de una variable proxy construida con la cantidad de ocupados en la filial. Dado que se trata de una variable continua, la evaluación de los efectos parciales se realiza a partir del Gráfico 1, donde puede observarse el cambio en la probabilidad de desarrollar uno u otro modelo a medida que aumenta el tamaño de la firma, dado el resto de las variables en sus niveles medios.

En términos generales, el Gráfico 1 permite concluir que, al aumentar el tamaño de las empresas, aumenta significativamente la probabilidad de pertenencia a modelos de mayor participación sindical. A su vez, también se destaca la influencia ejercida por el sector de actividad, ya que para aquellas EMN que se dedican a la actividad manufacturera la probabilidad de pertenecer al modelo de mayor participación es mayor al 60\%, aún para niveles bajos de ocupación. En cambio, para las EMN de los sectores de comercio y servicios la probabilidad de pertenecer a los clusters de menor participación es significativamente mayor y sólo al llegar a una cantidad de ocupados superior a 5.000 la situación cambia a favor del de mayor participación. 
Gráfico 1. Probabilidad de pertenencia a cada modelo de participación sindical, según el número de ocupados

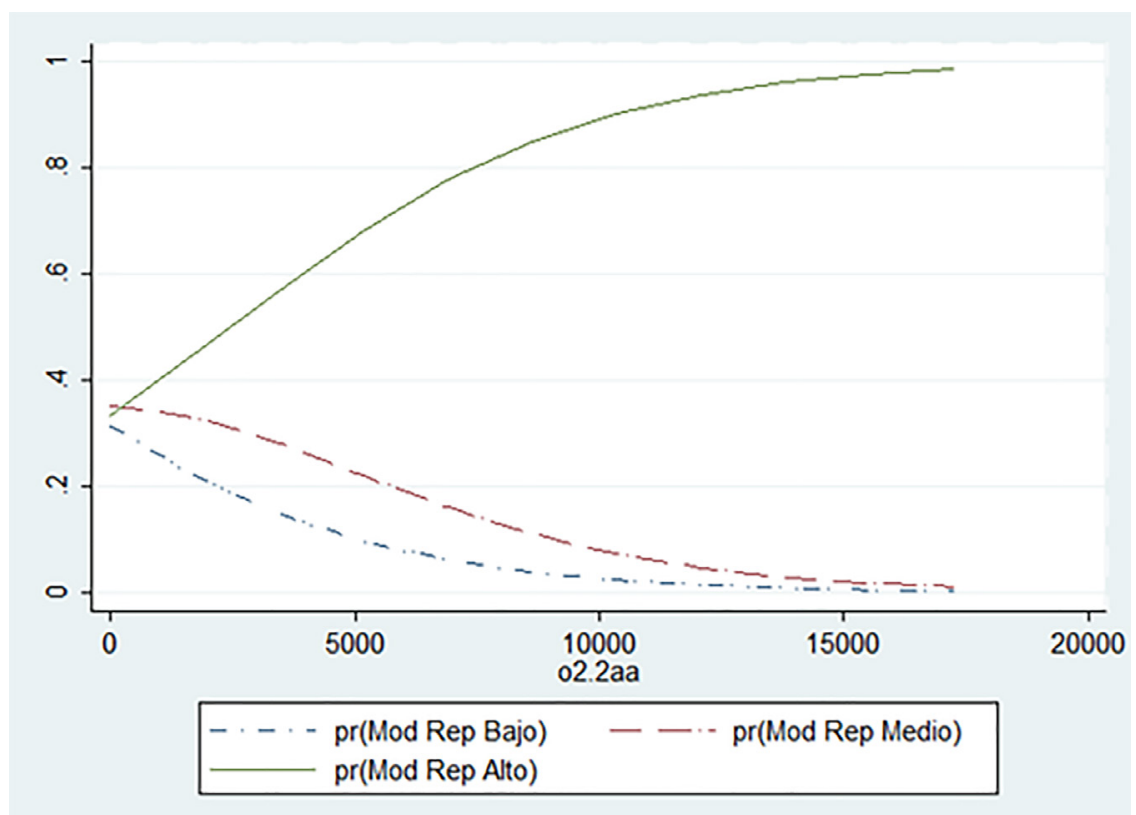

Fuente: Elaboración propia en base a encuesta a EMN.

\section{Discusiones y conclusiones}

Los estudios sobre EMN abordan distintas problemáticas desde diferentes puntos de vista, principalmente por la importancia que adquieren estas firmas en las economías e instituciones de los países en los que se radican. En este marco, este artículo se concentra en el estudio de las relaciones laborales en las filiales de Argentina y, más específicamente, de la complejidad que caracteriza a la relación entre las empresas y los sindicatos, teniendo en cuenta las lógicas internacionales que inciden sobre las filiales locales. Asimismo, se observa que las estrategias empresarias impactan sobre las instituciones y, a su vez, las instituciones condicionan las estrategias que buscan imponer estas empresas.

En el marco del relevamiento a filiales argentina de EMN realizado, este trabajo parte del análisis de los elementos que permiten desarrollar un modelo de participación específico en la empresa. Para ello se tuvo en cuenta la presencia sindical, la importancia de la afiliación y las características de los procesos de negociación que entablan los sindicatos con la empresa.

Considerando como referencia las hipótesis tradicionales sobre los determinantes de la participación sindical a nivel mundial en las EMN, se pudo identificaron lógicas que subyacen en las filiales argentinas de esas firmas. Para esto, se realizó un análisis de cluster que permitió establecer tres modelos de participación sindical en función de los tres ejes antes mencionados: nivel de sindicalización, presencia de delegados y política de negociación. Más allá de la fuerte presencia sindical que se verifica 
en las empresas instaladas en Argentina, lo cual está convalidado por los marcos institucionales, el análisis puso de manifiesto la existencia de formas antisindicales que se traducen en la importancia que alcanza el grupo de "baja participación" (35\% de los casos), el cual está definido por la ausencia de representación sindical en la firma y por la nula afiliación de los trabajadores al sindicato. En tanto, en el otro extremo, el $45 \%$ de las empresas se nuclean en el grupo de "alta participación".

Por su parte, el análisis realizado a partir del modelo logístico ordinal permitió corroborar las hipótesis relacionadas con la actividad, el tamaño, el año de ingreso al mercado local y la evaluación de desempeño. En este sentido, se ha destacado que i) las firmas industriales, ii) las de mayor tamaño relativo, iii) las que ingresaron al mercado local antes de 1990 y iv) las que no poseen sistemas de evaluación de desempeño no existen o estos son no individualizantes existe una mayor probabilidad de ubicarse en el grupo de alta participación sindical. En esta dirección, es posible sostener que las firmas que pertenecen al sector industrial y que cuentan con un mayor tamaño relativo poseen un arraigo sindical sustentado en la cultura, la historia y las instituciones locales. En esta misma lógica se inscriben las empresas que ingresaron al mercado argentino antes de 1990. Las formas de evaluación de desempeño de carácter individualizante tienen un lugar preponderante entre las firmas con menor participación sindical y socaban la idea, defendida por las organizaciones sindicales, de un mismo salario por la realización de tareas similares. Por el contrario, el salario basado en el desempeño hace mucho más heterogénea la relación anterior.

Estas evidencias se fortalecen al incorporar al análisis los efectos parciales y, con ello, la probabilidad de pertenencia de una filial a cada uno de los modelos de participación. En este caso, la mayor probabilidad de pertenecer al grupo de alta participación se evidencia entre aquellas empresas que poseen las cuatro características mencionadas, mientras que en el extremo opuesto, esto es, en i) las firmas que desarrollan actividades comerciales y/o de servicios, ii) que se instalaron después de 1990, iii) que son más pequeñas y que aplican evaluaciones de desempeño individualizantes, iv) las probabilidades mayores se observan en relación con la pertenencia al grupo de baja participación.

Finalmente, en lo que respecta a la relación entre el origen del capital de la EMN y la participación sindical (una de las principales vinculaciones retomadas en la literatura académica sobre este tema) no pudo comprobarse para el caso argentino una asociación significativa, aunque la tendencia observada se acerca a las hipótesis generalmente propuestas donde mayores niveles de participación sindical tienden a corroborarse entre las EMN que no son de Estados Unidos.

\section{Referencias bibliográficas}

Arrowsmith, J. y Marginson, P. (2011). Variable pay and collective bargaining in British retail banking. British Journal of Industrial Relations, 49 (1), pp. 54-79.

Barney, J (1991). Firm Resources and Sustained Competitive Advantage. Journal of Management, 17 (1), pp. 99-120.

Bechter, B., Brandl, B. y Meardi, G. (2012). Sectors or countries? Typologies and 
levels of analysis in comparative industrial relations. European Journal of Industrial Relations 18(3), pp. 185-202.

Bélanger, J., Giles, A. y Grenier, J. N. (2003). Patterns of corporate influence in the host country: a study of ABB in Canada' International Journal of Human Resource Management, 14(3), pp. 469-85.

Cooke, W. (2003). The influence of industrial relations systems factors on foreign direct investment. En Cooke, W. (Ed.) Multinational Companies and Global Human Resource Strategies. Westport, EEUU: Quorum Books.

Charlwood, A. y Haynes, P. (2008) Union Membership Decline in New Zealand, 1990-2002. Journal Industrial relations, 50(1), pp. 87-110.

Crivisqui, E. (1993). Análisis factorial de correspondencias. Asunción, Paraguay: Editorial del Laboratorio de Informática de la Universidad Católica de Asunción.

Cruz Villalón, Jesús (2003). Las relaciones laborales en la pequeña empresa: una aproximación a sus especialidades normativas. En Cruz Villalón, J. Las relaciones laborales en la pequeña empresa. Universidad de Cadiz.

Delfini, M. y Roitter, S. (2007). Las relaciones laborales en una trama automotriz argentina. Cuadernos de Relaciones Laborales, 25(1), pp. 195-221.

Delfini, M. y Erbes, A. (2011). La gestión de la fuerza de trabajo en las filiales argentinas de Empresas Multinacionales. En Novick, M., Palomino, H. y Gurrera, M. S. (Coords.), Multinacionales en la Argentina. Estrategias de empleo, relaciones laborales y cadenas globales de valor. Buenos Aires, Argentina: Ministerio de Trabajo, Empleo y Seguridad Social, Programa de las Naciones Unidas para el Desarrollo.

Delfini, M., Erbes, A. y Gurrera, M. S. (2011). Estrategias gerenciales de las filiales Argentinas hacia los empleados y sus representantes. En Novick, M., Palomino, H. y Gurrera, M. S. (Coords.), Multinacionales en la Argentina. Estrategias de empleo, relaciones laborales y cadenas globales de valor. Buenos Aires, Argentina: Ministerio de Trabajo, Empleo y Seguridad Social, Programa de las Naciones Unidas para el Desarrollo.

Delfini, M. (2011). Relaciones laborales y "gestión de recursos humanos" en filiales de Empresas Multinacionales en Argentina. Revista Sociedad y Economía, 20, 149-170.

Edwards, T., Ress, C. y Coller, X. (1999). Structure, Politics and the Diffusion of Employment Practices in Multinationals. European Journal Industrial relations, 5(3), pp. 286-306.

Edwards, P., Edwards, T., Ferner, A., Marginson, P. y Tregaskis, O. (2007). Employment Practices of MNCs in Organisational Context: A Large-Scale Survey. Recuperado de: http://www.colef.mx/jorgecarrillo/multinacionales/ 
Edwards, T. y Kuruvilla, S. (2005). International HRM: National business systems, organizational politics and the international division of labour in MNCs. Recuperado de: http://digitalcommons.ilr.cornell.edu/articles/169/

Edwards, T., Marginson, P. y Ferner, A. (2013). Multinational companies in crossnational context: integration, differentiation and the interactions between MNCs and nation states. Industrial and Labor Relations Review, 66(3), pp. 547-587.

Fernández Macías, E. (2004). Nuevos tiempos de trabajo y calidad del empleo. En Esteve Mora, F., Fernández Macías, E., García Laso, A. y Muñoz de Bustillo Llorente, R. (Dirs.), Nuevos tiempos de actividad y empleo. Salamanca, España: Ministerio de trabajo y asuntos sociales.

Ferner, A. y Almond, P. (2013). Performance and reward practices in foreign multinationals in the UK. Human Resource Management Journal, 23(3), pp. 241-261.

Ferner, A., Edwards, T. y Tempel, A. (2011). Power, institutions and the cross-national transfer of employment practices in multinationals. Human relations, 65(2), pp. 163-187.

Flood, P. y Toner, B. (1997). Large non-union companies: How do they avoid a catch 22. British Journal of Industrial Relations, (35), pp. 257-77.

García Calavia, M. (2001) El sindicalismo tras la reestructuración productiva. Papers: Revista de Sociología, (65), pp. 11-30.

Gunnigle, P., Turner, T. y D'Art, D. (1998). Counterpoising Collectivism, PerformanceRelated Pay and Industrial Relations in 'Greenfield'. British Journal of Industrial Relations, 36(4), pp. 565-579.

Gunnigle, P., Lavelle, J. y McDonnell, A. (2007). Industrial relations in MNC: doublebreasting and trade union avoidance in Ireland. Working Paper, Department of Personnel and Employment Relations, Kemmy Business School, University of Limerick, National Technological Park, Limerick, Ireland.

Gunnigle, P. (1995). Collectivism and the Management of Industrial Relations In Greenfield Sites. Human Resource Management Journal, 5(3), pp. 24-40.

Haberfeld, Y. (1995). Why do workers join unions? The case of Israel. Industrial and Labor Relations Review, 48(4), pp. 656-670.

Harzing, A. (1999). Managing the Multinationals: An international study of control mechanisms. Cheltenham, Inglaterra y Northampton, EEUU: Edward Elgar Publishing Limited.

Harzing, A. (2001). An analysis of the functions of international transfer of managers in MNCs. Employee Relations, 23(6), pp.581-598.

Heery, E. (1997). Performance-related pay and trade union de-recognition. Employee Relations, 19(3), pp. 208-221. 
Heery, E. (2000). Trade unions and the management of reward. En White, G. y Druker, J. (Eds.), Reward Management. A Critical Text. Londres, Inglaterra: Routledge.

Hyman, R. (1996). Los sindicatos y la desarticulación de la clase obrera. Revista Latinoamericana de Estudios del Trabajo, 2(4), pp. 10-23.

Hyman, R. (1999). National Industrial Relations Systems and Transnational Challenges: An Essay in Review. European Journal of Industrial relations, 5(1), pp. 89-110.

Jacoby, S. (1985). Employing Bureaucracy Managers, Unions, and the Transformation of Work in the 20th Century. Londres, Inglaterra: Lean.

Katz, H. y Darbishire, O. (2000). Converging Divergences. Cornell University Press.

Kristensen, P. y Zeitlin, J. (2005). Local Players in Global Games: The Strategic Constitution of a Multinational Corporation. Oxford, Inglaterra: Oxford University Press.

Linhart, D. (1997). La modernización de las empresas. Buenos Aires, Argentina: CEIL-PIETTE.

Machin, S. (2004). Factors of Convergence and Divergence in Union Membership. British Journal of Industrial Relations, 42(3), pp. 423-438.

Marginson, P., Arrowsmith, J. y Gray, M. (2008). Undermining or reframing collective bargaining? Variable pay in two sectors compared. Human Resource Management Journal, 18(4), pp. 327-346.

Marginson, P. y Sisson, K. (2004). European Integration and Industrial Relations. Basingstoke, Inglaterra: Palgrave/Macmillan.

Marshall, A. y Groisman, F. (2005). Sindicalización en la Argentina: Análisis desde la perspectiva de los determinantes de la afiliación individual. En $7^{\circ}$ Congreso nacional de ASET. Buenos Aires, Argentina.

Martinez, I. y Jarrillo, C. (1989). The Evolution of Research on Coordination Mechanisms in Multinational Corporations. Journal of International Business Studies, 20(3), pp. 489-514.

Meardi, G. (2004). Short circuits in multinational companies: the extension of European Works Councils to Poland. European Journal of Industrial Relations, 10( 2), pp. 161-178.

Novick, M., Palomino, H. y Gurrera, M. S. (Coords.). (2011). Multinacionales en la Argentina. Estrategias de empleo, relaciones laborales y cadenas globales de valor. Buenos Aires, Argentina: Ministerio de Trabajo, Empleo y Seguridad Social, Programa de las Naciones Unidas para el Desarrollo.

Roitter, R. y Erbes, A. (2017). La importancia de las empresas multinacionales en el empleo. Evidencia reciente sobre Argentina (1990-2013). En Ventricci, P., 
Rodriguez Miglio, M., Pinazo, G. y Bosisio, W., El trabajo y las empresas multinacionales hoy: economía, relaciones laborales y conflictividad. Los Polvorines, Argentina: UNGS.

Roitter, S. (1991). Análisis factorial de correspondencias múltiples. Córdoba, Argentina: Mimeo UNC.

Riley, M. (1997). Determinants of Union Membership: A Review. Labour, 11(2), pp. 265-301.

Royle, T. (1998). Avoidance Strategies and the German System of Co-determination. International Journal of Human Resource Management, 9(1), pp. 1026-1047.

Tempel, A. (2001). The Cross-National Transfer of Human Resource Management Practices in German and British Multinational Companies. Mering, Alemania: Hampp.

Trebilcock, A. (1998). Relaciones laborales y gestión de recursos humanos: una vision general. En OIT [Organización Internacional del Trabajo], Enciclopedia de salud y seguridad en el trabajo. Ginebra, Suiza: OIT.

UNCTAD [Conferencia de las Naciones Unidas sobre Comercio y Desarrollo]. (2016), World Investment Report 2008 - Transnational Corporations and the Infrastucture Challenge, Nueva York, EEUU: Naciones Unidas.

Van Het Kaar, R. H. y Grünell, M. (2001). Variable pay in Europe. EIR Observer, 3(1), pp. 1-8. 